\title{
Pharmacological management of core and comorbid symptoms in autism-spectrum disorder
}

\author{
Rachel Elvins \& Jonathan Green
}

\begin{abstract}
SUMMARY
In this article we review the evidence base and practical experience supporting the use of psychopharmacological treatments for autism-spectrum disorders across the lifespan. Recent advances in knowledge are highlighted, as are the shortcomings of published data in this field. Psychosocial and educational interventions remain the core of treatment, but there is now evidence that medication management is a good option for certain domains of behaviour. We discuss the evidence in terms of symptom clusters, including the underlying social deficit of autism and common psychiatric comorbidities.
\end{abstract}

\section{DECLARATION OF INTEREST}

The authors have attended educational events sponsored by Eli Lilly and Janssen-Cilag.

Autism-spectrum disorders are one of the leading causes of lifetime developmental disability (Fombonne 2003). These diagnoses are often grouped as pervasive developmental disorders and include childhood autism, atypical autism and Asperger syndrome. They are recognised as complex neurodevelopmental disorders, often becoming clinically apparent in the second to third year of life. The diagnosis is based on disturbance in the domains of: reciprocal social interactions, communication, restricted interests and stereotyped patterns of behaviour (World Health Organization 1994). Up to two-thirds of affected individuals will present with a degree of global intellectual disability, although some may have a very uneven profile of abilities. Accurate diagnosis, usually made by a combination of direct observation of behaviour and informant history, is complicated by considerable heterogeneity in the manifestation of these core deficits, by variation in ability level and by developmental changes. However, it is clear that autism-spectrum disorders persist across the lifespan, with varied complex needs in adult life (Lord 2001). The course of development into old age is, as yet, largely unknown.
Prevalence figures vary widely depending on the definition of caseness and exact assessment tools used. Until recently, prevalence figures of 0.3-0.9\% were reported for all autism-spectrum disorders (Fombonne 2005). Latterly, UK studies have reported a higher prevalence of around 1.2\%, which probably reflects better ascertainment of a broader phenotype (Baird 2006). There are no accurate prevalence figures for adults with autism. A male excess of between 3:1 and 4:1 is generally observed (Fombonne 2003).

Maladaptive behaviours and comorbid psychiatric symptoms are common in individuals with autismspectrum disorders and are strongly associated with caregiver stress (Lecavalier 2006).

Autism is thus a relatively common, chronic, potentially substantially disabling disorder, with significant costs to both the affected individual and to family members. There are no established definitive treatments for the core social impairment. Both the severity of the disorder and lack of effective treatments continue to promote keen public interest and prompt research. Controversies surrounding environmental causes of autism and novel unproven treatments serve to keep this set of disorders in the public eye and increase referrals to both paediatric and psychiatric services. Since the introduction of the National Autism Plan for Children in the UK (National Initiative for Autism: Screening and Assessment 2003), there has been a further focus on early intervention as well as assessment. More people are now first diagnosed with autism as adolescents and adults, possibly owing to increased public and professional recognition (Mesibov 1997). For many families, the transition from adolescence to adulthood will be a period of increased need. As a result, a greater number of referrals may be seen across adult psychiatry.

\section{Medication management in autism}

Alongside burgeoning professional interest, increasingly rigorous clinical trial methodology has attempted to focus on evidence-based pharmacological strategies for both the core
Rachel Elvins is a clinical tutor in child and adolescent psychiatry at the School of Psychiatry, University of Manchester. She is also an honorary specialist registrar in child and adolescent psychiatry at the Central Manchester Foundation Trust. Her main interests and research are in the therapeutic alliance in treatment trials and interventions in autism-spectrum disorders. Jonathan Green is Professor of Child Psychiatry at the University of Manchester. He is also an honorary consultant psychiatrist at the Central Manchester Foundation Trust. His interests include early intervention in autismspectrum disorders, attachment disorder and the therapeutic alliance.

Correspondence Rachel Elvins, Psychiatry Research Group, 4th Floor (East), University Place, University of Manchester, Oxford Road, Manchester M13 9PL, UK. Email: rachel.elvins@manchester.ac.uk 
tPsychological interventions for autism-spectrum disorders in childhood are discussed in Advances in Howlin P (2010) Evaluating psychological treatments for children with autism-spectrum disorders. 16: 133-140. Ed. syndrome and comorbidity in autism-spectrum disorders. In the field of neurodevelopmental disability there has traditionally been a dearth of independent, well-powered randomised controlled trials (RCTs). However, more rigorous psychopharmacology studies have recently started to be published. Educational and psychosocial interventions are the mainstay of treatment, with the aim of improving language acquisition and maximising communication and social skills. ${ }^{\dagger}$ However, medication management, particularly of maladaptive behaviours, is common and possibly increasing (Aman 2005). Such behaviours or symptoms of comorbid disorders may interfere with socialisation and educational progress, and severely impair individuals' and families' quality of life. Up to $75 \%$ of individuals with autism and intellectual disabilities were prescribed at least one psychotropic medication in a recent UK study (Tsakanikos 2006). Factors associated with increasing medication use include greater age, poorer functioning and higher levels of challenging behaviour (Aman 2005).

There are, however, no currently available standard medication regimes. Medications that are commonly used belong to diverse groups and are non-specific to the target symptoms identified. They may affect a wide range of neurological functions, with subsequent unwanted effects. An attempt to elucidate the evidence base for the use of such treatments is therefore helpful for prescribers and case managers alike.

The aim of this paper is to discuss and summarise clinical pharmacotherapy for autism-spectrum disorders based upon target domains of behaviour (Table 1). Such symptoms, which are difficult to manage, are often the reason that families seek medical treatments.

We first evaluate treatments targeted at the underlying core social deficit of autism and then address certain target clusters of symptoms such as stereotypical and compulsive/ritualistic behaviours, and serious aggressive and selfinjurious behaviour. The distinction between core syndrome phenomena and co-occuring symptoms is important in assessment and treatment planning, even though this distinction is sometimes rather theoretical: improvement in one area may lead to global improvements, and some drugs may play a role in several symptom clusters. However, a systematic approach that makes this distinction will help clinicians organise their thinking when

\section{TABLE 1 Selected medications for use in domains of behaviour in autism}

\begin{tabular}{|c|c|c|c|}
\hline Symptom cluster & Medications & Common/important adverse effects & Supporting references \\
\hline \multirow[t]{2}{*}{$\begin{array}{l}\text { Stereotypical and } \\
\text { compulsive/repetitive } \\
\text { behaviours }\end{array}$} & SSRI (fluoxetine, fluvoxamine) & $\begin{array}{l}\text { Irritability, activation, insomnia, } \\
\text { gastrointestinal upset, potential increase in } \\
\text { suicidal ideation }\end{array}$ & $\begin{array}{l}\text { McDougle 1996; Hollander 2005; Posey } \\
2006\end{array}$ \\
\hline & Atypical antipsychotic (risperidone) & $\begin{array}{l}\text { Appetite increase, weight gain, sedation, } \\
\text { glucose dysregulation, extrapyramidal } \\
\text { symptoms, QTc prolongation, neuroleptic } \\
\text { malignant syndrome }\end{array}$ & Scahill 2002; McDougle 2005 \\
\hline \multirow{3}{*}{$\begin{array}{l}\text { Irritability, aggression } \\
\text { and self-injury }\end{array}$} & Atypical antipsychotic (risperidone) & As above & McDougle 1998; Shea 2004 \\
\hline & Opiate antagonist (naltrexone) & Headaches and dizziness & Symons 2004 \\
\hline & Typical antipsychotic (haloperidol) & $\begin{array}{l}\text { Extrapyramidal symptoms, akathisia, weight } \\
\text { gain, QTc prolongation, neuroleptic malignant } \\
\text { syndrome }\end{array}$ & Remington 2001 \\
\hline \multirow[t]{3}{*}{$\begin{array}{l}\text { Hyperkinesis and } \\
\text { inattention }\end{array}$} & Immediate-release methylphenidate & $\begin{array}{l}\text { Appetite suppression, weight loss, insomnia, } \\
\text { rebound hyperkinesis, tachycardia, } \\
\text { hypertension }\end{array}$ & $\begin{array}{l}\text { Research Units on Paediatric } \\
\text { Psychopharmacology Autism Network } \\
\text { 2005a }\end{array}$ \\
\hline & Alpha-2 agonist (clonidine) & Fatigue, hypotension & Jaselskis 1992 \\
\hline & Atomoxetine & $\begin{array}{l}\text { Gastrointestinal upset, fatigue, appetite } \\
\text { suppression, liver dysfunction, suicidal } \\
\text { ideation, hypertension, tachycardia }\end{array}$ & Arnold 2006 \\
\hline Depressive symptoms & SSRI & As above & Posey 2006 \\
\hline Manic symptoms & $\begin{array}{l}\text { Anticonvulsant mood stabiliser } \\
\text { (valproate/divalproex) }\end{array}$ & $\begin{array}{l}\text { Weight gain, sedation, gastrointestinal } \\
\text { upset, platelet suppression, liver dysfunction, } \\
\text { pancreatitis, teratogenicity }\end{array}$ & Hollander 2001 \\
\hline \multirow[t]{2}{*}{ Anxiety symptoms } & SSRI & As above & Posey 2006 \\
\hline & Buspirone & Dyskinesia & Buitelaar 1998a \\
\hline \multirow[t]{2}{*}{ Sleep dysfunction } & Melatonin & Somnolence & Owens 2005 \\
\hline & Antihistamine & Irritability, somnolence & Owens 2005 \\
\hline
\end{tabular}

SSRI, selective serotonin reuptake inhibitor. 
deciding on a particular medication strategy, allowing them to focus on exactly which presenting problem they are trying to treat, and with what. A methodical approach of this kind is also essential to minimise polypharmacy and the development of potentially disastrous management strategies in which further medication has to be used to treat emerging unwanted effects of previous medications.

We will focus on RCTs, where available, as well as common unwanted effects of particular drugs and shortcomings of the published data. Although the field is not yet at a stage at which treatment protocols or algorithms could usefully be written, the aim will be to identify medication regimes which are most likely to be clinically useful for each domain. Medication management of common psychiatric comorbidities in autism will also be discussed.

Finally, future directions of pharmacotherapeutic research will be highlighted.

\section{Core autism-spectrum disorder symptomatology}

\section{Social deficits}

The detailed underlying pathophysiology of social impairments in autism-spectrum disorders is still unclear. Their treatment has been subject to many 'false dawns' in the literature, with therapeutic approaches often based on questionable theory. Some rigorous trials have on occasion reduced interest in unproven approaches despite their active promotion by vested interests. Most recently, secretin, a gastrointestinal peptide, and fenfluramine, an indirect 5-HT partial agonist, have been widely trialled, with nearly 500 children with autism-spectrum disorders being recruited to RCTs for secretin alone. Both drugs were shown in preliminary trials to have prosocial effects in autism. However, placebo-controlled trials have failed to show consistent improvements (McDougle 2006). Neither medication has any evidence base in autism management at this time.

\section{Diet}

Vitamins and minerals have been widely trialled, with broadly disappointing results, a recent example being a Cochrane review of RCTs studying the use of vitamin B6 and magnesium (Nye 2005). It was concluded that their use cannot be supported in autism-spectrum disorders based on the available evidence, and that larger, better designed studies are needed. There is widespread interest in exclusion diets, but as yet there have been no good-quality RCTs in this area.

\section{Immune function}

Infectious and immune mechanisms have been popular candidate aetiological agents in autism (although there is no significant support for this from basic science). A limited number of related treatment studies have been carried out. One trial of vancomycin (Sandler 2000) showed that improvements in communication returned to baseline when the drug was stopped. Systematic RCTs of drugs having a direct effect upon immune function in autism have not so far been conducted.

There is, at present, no place for immunotherapy of any kind in the management of autism. There is also no reliable evidence that anti-fungal treatments are effective in the treatment of autismspectrum disorders.

Vaccinations, particularly the combined measles, mumps and rubella (MMR) vaccine, have long been the subject of controversy with regard to aetiological mechanisms and autismspectrum disorders. Several large-scale studies have subsequently not supported a causal link between MMR and autism (Honda 2005).

\section{Antidepressants and antipsychotics}

Open-label reports of selective serotonin reuptake inhibitors (SSRIs) and atypical antipsychotics such as risperidone have suggested that some individuals with autism show improvement in aspects of social relatedness following treatment (McDougle 2006). This has yet to be consistently established in RCTs (Hollander 2005), but some clinicians would support their empirical use. Risperidone does have a proven effect on arousal states and behaviour (see below), and any effect on prosocial behaviour is likely to be secondary to this.

\section{Glutamate-active medication}

Glutamatergic function has been the focus of recent extensive research in neuropsychiatric disorders. Glutamate is the primary excitatory amino acid in the brain and is thought to be important in regulating neuronal plasticity and higher cognitive functions (Carlsson 1998). Both autism-spectrum disorders and schizophrenia are postulated to be hypoglutamatergic disorders and parallels have been drawn between the negative symptoms of schizophrenia and the social impairment in autism (Nikolov 2006).

Further interest has been excited by a recent large pooled genetic analysis (Autism Genome Project Consortium 2007) which used linkage and copy number variation analysis respectively to implicate candidate gene loci on chromosome 
11p12-p13 and neurexins. Neurexins and neuroligins (independently linked with autism in other analyses) are implicated in glutamatergic synaptogenesis, highlighting glutamate-related genes as promising candidates in autism-spectrum disorders. One glutamate-active drug of interest is D-cycloserine (a partial agonist at the $N$-methylD-aspartic acid (NMDA) receptor complex). It has been used with benefit when added to conventional antipsychotics in schizophrenia and this benefit has been associated with enhanced temporal lobe function (Yurgelun-Todd 2005). A small singleblind pilot study of D-cycloserine targeting the core social impairment in children with autismspectrum disorders (Posey 2004) showed greater improvements on the Clinical Global Impressions (CGI) scale and the Aberrant Behaviour Checklist $(\mathrm{ABC})$ social withdrawal scale, with ascending dosages. Adverse effects were reported only at higher doses. However, Posey recently reported a larger, double-blind parallel groups study which showed that D-cycloserine did not improve social withdrawal in young children (Posey 2008). Additional subgroup analyses are planned to determine whether individual characteristics are associated with response. Other drugs currently being trialled include amantadine and memantine, which act as non-competitive antagonists at the NMDA receptor.

One small controlled trial of amantadine in children (King 2001) showed a trend towards greater treatment response based on CGI ratings. Other studies have been less optimistic, for example lamotrigine (an anticonvulsant which attenuates cortical glutamate release) was found to be no better than placebo on any outcome measure employed in a small RCT in children (Belsito 2001). The active research interest in this area parallels that in schizophrenia and more work in this area will undoubtedly follow. However, the work is at a preliminary stage and at present the available evidence would not support the clinical use of glutamate-active medications in autism.

\section{Clinical implications}

The core social impairments in autism-spectrum disorders remain relatively intractable. In childhood and adolescence, psychosocial and education management strategies should be the first line in management and do have an evidence base for efficacy in some situations. The current lack of convincing evidence for a pharmacological approach to long-term disabilities means that at present medication management does not have a significant place in practice. Future advances may change the situation.

\section{Stereotypical and ritualistic/compulsive behaviours}

These behaviours are defined as part of the core of autism. They include verbal and motor rituals, obsessive questioning, rigid adherence to routine, preoccupation with details and obsessive desire for maintenance of sameness. Such symptoms are commonly the most functionally impairing aspect of the syndrome and interfere significantly with an individual's progression in educational and socialisation programmes. They have similarities with obsessive-compulsive disorder (OCD) phenomena and thus medications such as tricyclic antidepressants and SSRIs with known efficacy in OCD are obvious candidate drugs.

\section{Antidepressants}

Clomipramine is a non-selective tricyclic antidepressant which affects uptake of serotonin, noradrenaline and dopamine. Two crossover RCTs using children and young adults provide some evidence that clomipramine is superior to placebo on measures such as anger and obsessive symptoms. However, there were no gains for areas such as hyperactivity and global symptom severity (Remington 2001). Concerns about adverse effects of clomipramine make this drug less widely used than SSRIs.

Using the SSRI fluvoxamine, a short-term RCT in adults with autism showed improvements in both compulsive and prosocial behaviour (McDougle 1996). Trials with children have had more mixed results. A short-term RCT involving children with autism-spectrum disorders showed that fluvoxamine had no advantage over placebo (McDougle 2000). Adverse effects were noted in $78 \%$. More encouragingly, another 12 -week RCT crossover study of 18 children with autismspectrum disorders judged that 10 were treatment responders on the CGI. However, a placebo response rate is not recorded, and adverse events occurred in 39\%, although this was not considered significant (Sugie 2005).

Improvements in repetitive behaviours have been shown in two very small crossover RCTs in children and adults using fluoxetine (Buchsbaum 2001). There were no differences in side-effects between drug and placebo in children (Hollander 2005). Unpublished data of a recent large-scale $\mathrm{RCT}$ of fluoxetine in children showed that repetitive behaviours were reduced in those taking either placebo or fluoxetine but there were no statistically significant differences in response between the two groups. Full analysis of the trial is ongoing to establish the reasons for this unexpected result (Autism Speaks 2009). 
Evidence regarding other antidepressant use is scant and of less quality. Open-label trials of sertraline, citalopram and escitalopram in children and adults have showed benefits in reducing aggression and repetitive behaviours (e.g. Owley 2005). An open-label study using paroxetine in adults showed improvements at 1-month but not at 4-month follow-up (Davanzo 1998). There are no studies using paroxetine in children as yet. A recent large-scale RCT of citalopram in children with autism-spectrum disorders and repetitive behaviours showed no significant differences in response between the citalopram- and placebotreated group, although both groups showed improvements. Citalopram was more likely to be associated with adverse events such as increased energy levels and insomnia (King 2009). Openlabel studies of venlafaxine have suggested efficacy in repetitive behaviours in some children and adults (Hollander 2000).

The studies above may indicate that SSRIs may be more effective and give rise to fewer side-effects in adolescents and adults with autism-spectrum disorders than in children (Erickson 2007).

\section{Antipsychotics}

Two large multisite trials in children (Shea 2004; McDougle 2005) showed that risperidone was significantly more effective than placebo for reducing interfering behaviours. However, in smaller RCTs with preschool age children with autism, risperidone was not meaningfully superior to placebo (Luby 2006). Adverse effects of atypical antipsychotics include increased appetite and weight gain, dyslipidaemia and insulin resistance, somnolence, extrapyramidal symptoms and prolactin elevation. One study looking at prescribing of risperidone over the course of 12 months used a pooled database of prolactin levels in 700 children (Dunbar 2004). Mean levels were found to increase and peak in the first 1-2 months and then return to near normal by 3-5 months. There was no associated delay in growth or sexual maturation.

Prolongation of the QTc interval with risperidone has been reported, but studies in both adults and children did not find prolongation beyond the threshold accepted as being associated with torsade de pointes or other significant electrocardiogram (ECG) changes (e.g. Harrigan 2004).

\section{Clinical implications}

In this domain of core autism symptomatology, the situation is rather the reverse of the social deficits. Psychosocial or cognitive-behavioural interventions can be useful adjuncts but may be less effective in this patient group than they can be, for example, in people with autism and comorbid obsessive-compulsive symptoms. Medication management has some evidence base and clinical experience suggests it can be useful. Medication effects, however, do show a great deal of individual variation, and patients and families need to understand that trials of medication may be exploratory and be subject to careful monitoring and dosage adjustment. A recent review (McDougle 2006) suggests that, given the side-effect profile of risperidone, low-dose SSRIs should be used as first-line treatment if repetitive behaviour is the main focus of treatment. However, even the evidence base for SSRIs consists of small shortterm RCTs with heterogenous populations and varying outcome measures.

\section{Co-occurring or comorbid psychiatric symptoms}

Autism-spectrum disorders have a high prevalence of both psychiatric and physical comorbidities (Broadstock 2007). These tend to emerge in middle childhood, and wax and wane according to circumstance. At a theoretical level it is not always clear that such symptoms constitute a true psychiatric comorbidity; commonly, symptoms will co-occur with autism because of the interaction between the developmental disorder and concurrent stressors such as increased social demands, inadequately adapted schooling, a sense of rejection or poor self-image, bullying or family conflict. These symptoms are important to understand since they are often as functionally impairing and yet more tractable than the core disorder, and are often a key target for intervention.

Accurate diagnosis of comorbid psychiatric disorders can be difficult. Modifications of diagnostic criteria may be necessary to account for differing clinical presentations in individuals with developmental disability. 'Diagnostic overshadowing' may prevent accurate detection of symptoms; even if detected, they may be spuriously attributed to the core disorder (Dykens 2000). It is also clear, however, that a condition like autism will be associated with additional symptoms which may not rise to the level of 'disorder'. Deciding when and whom to treat may be largely based on functional impairment. Conditions such as depression and anxiety (as opposed to the core syndrome) may be eminently treatable and tackling them may vastly improve patients' and families' quality of life.

A growing body of literature is focused on the evidence base for treating symptoms of hyperkinetic disorder in autism. However, there is a relative dearth of high-quality data considering other psychiatric comorbidities. Most of the 
available data come from trials designed to assess core and behavioural symptoms associated with autism, but which also found improvements in other domains such as depressive or anxious symptoms. Randomised controlled trials studying patients with both autism-spectrum disorders and adequately defined psychiatric comorbidities are urgently needed, but are difficult to design and carry out. Much of the data on psychiatric comorbidity in adolescents and adults are based on case reports and are difficult to interpret given the potential for selection, referral or reporting biases.

\section{Maladaptive aggression and self-injury}

Self-injurious behaviour and aggression can severely disrupt the management of autism. Many pharmacological treatments have been trialled.

\section{Antipsychotics}

Atypical antipsychotics are the most frequently used psychotropic medication for aggression and serious self-injury in people with autism (Erickson 2007). Moderately sized RCTs of risperidone in both adults and children (Shea 2004) have described beneficial effects. Open-label studies with a double-blind discontinuation component have suggested both longer-term benefits and tolerance (e.g. Research Units on Paediatric Psychopharmacology 2005b). In 2006, the US Food and Drug Administration approved risperidone for the symptomatic treatment of irritability (including aggression and self-injury) in children and adolescents. In the UK, a Cochrane review (Jesner 2007) concluded that risperidone can be beneficial, but that the lack of a single standardised outcome measure did not allow direct comparison of studies.

Evidence of efficacy of other atypicals is very preliminary. One small RCT of olanzapine found it to be effective in about $50 \%$ of children (Hollander 2006a). However, olanzapine is strongly associated with weight gain and other physical morbidity. Open-label trials of quetiapine indicate that the response rate and tolerability are poor (e.g. Martin 1999). Clozapine and ziprasidone are rarely used because of the risk of blood dyscrasias and QTc prolongation respectively. Open-label trials of aripiprazole in children with autism-spectrum disorders have been encouraging, and seem to be well tolerated (Stigler 2009). An RCT in children with autism-spectrum disorders is currently ongoing in the USA (Erickson 2007).

Several large, well-designed RCTs (e.g. Remington 2001) have studied haloperidol and found it efficacious in both children and adults with autism and behaviour problems. Adverse events including dyskinesia and sedation are common, however, and it is therefore more often reserved for treatment-refractory symptoms.

\section{Opiate antagonists}

Initial findings of open-label studies of naltrexone seemed promising (Panksepp 1991), but subsequent placebo-controlled studies showed no positive effects for the core social deficits. The most consistent finding is a modest reduction in hyperactivity (Feldman 1999). A quantitative review of the literature suggests that naltrexone might be beneficial for reducing self-injurious behaviours in individuals with mental retardation, including those with autism-spectrum disorders (Symons 2004).

\section{Mood stabilisers}

Evidence regarding the effects of anti-epileptic medication is mixed. A small RCT of valproate (Hellings 2005) involving 30 adolescents could not demonstrate a significant difference between the drug and placebo, but a small trial of 13 children treated with divalproex (Hollander 2006b) demonstrated benefits.

Published data for carbamazepine are limited to case reports. Hepatotoxicity, weight gain, sedation and teratogenicity are important side-effects for this group of drugs. There are no controlled lithium trials in autism-spectrum disorders. Evidence for alpha-2 agonists such as clonidine and beta blockers are limited to small RCTs and case reports (Myers 2007).

\section{Clinical implications}

Severe maladaptive aggression and self-injury can be the most disturbing and impairing of cooccurring symptoms in autism, particularly in individuals with intellectual disability. Medication management has a clear role here. Antipsychotics, particularly risperidone, have a consistent evidence base, are increasingly prescribed and can be very useful. However, medication management should be done carefully and well monitored; doses should be titrated up from a low base to the 'minimum effective dose' using frequent detailed symptom monitoring. In this way the emergence of medication side-effects (so easily confused with the target symptoms themselves) can be identified and the real effect of the drug can be assessed. Another reason for detailed assessment and targeting of symptoms is that families may be unrealistic about the limitations of medication in complex situations; the placebo response in parental report is often strong. Specific medications should not be pursued if there is no evidence of their benefit; but 
withdrawal should be slow and judicious to avoid withdrawal effects. The limitations of published studies and drug side-effect profiles should be carefully considered; however, judged carefully and used with persistence, medication management can transform children's development and families' lives.

\section{Hyperkinesis and inattention}

Symptoms of hyperkinetic disorder (inattention, impulsivity, distractibility and hyperactivity) are very common in autism, particularly in children and adolescents (Lee 2006). Traditionally in UK diagnostic schemes, a diagnosis of hyperkinetic disorder is not made if it occurs exclusively during the course of an autism-spectrum disorder (the diagnostic hierarchy concept). However, this has the limitation of tending to underplay or even obscure significant and treatable symptom cooccurrence. These symptoms may severely impair an individual's functioning and warrant treatment in their own right. ICD-11 is likely to relax this strictly hierarchical approach to diagnostic formulation.

\section{Psychostimulants}

Randomised controlled trials of methylphenidate have demonstrated improvement in childhood autism (Quintana 1995; Research Units on Paediatric Psychopharmacology Autism Network 2005a). This applies to immediate-release preparations but it is unclear whether the results can be applied to other stimulants. Trials suggest that the response rate in people with autism is lower than that of people without autism, and that side-effects such as irritability and poor appetite are more common (Erickson 2007). One trial suggested that patients with Asperger syndrome may respond more positively than those with other autism-spectrum disorders, but not better than neurotypical individuals (Stigler 2004). However, a more recent, large open-label study found no statistically significant difference in degree of response or adverse events between those with autism-spectrum disorders and those without (Santosh 2006). Secondary analysis of the Research Units on Paediatric Psychopharmacology Autism Network (2005a) trial also suggested that psychostimulants may have a positive effect on some aspects of social communication in children with autism-spectrum disorders and hyperactivity, such as joint attention initiations (Jahromi 2009).

\section{Atomoxetine}

Atomoxetine is a selective noradrenaline inhibitor. Recently, open-label studies and a small pilot RCT (e.g. Arnold 2006) have suggested effectiveness for hyperactivity, impulsivity and oppositional behaviour in autism-spectrum disorders. Common side-effects include nausea, increased heart rate and fatigue.

\section{Alpha-2 adrenergic agonists}

Two very small RCTs involving a total of 17 patients (Fankhauser 1992) have suggested modest benefits of clonidine, and one small crossover study suggests similar effects for lofexidine (Niederhofer 2002). One open-label trial indicates that guanfacine may also be helpful for hyperactivity (Scahill 2006). Common side-effects include sedation and hypotension.

\section{Cholinergics}

Open-label trials and case series of the acetylcholinesterase inhibitors donepezil, galantamine and rivastigmine have reported improvements in hyperactivity, inattention and irritability (Chez 2004).

\section{Amantadine}

One RCT of amantadine for children (Ing 2001) showed some improvements in hyperactivity and impulsivity on observer measures but not on parent-scored measures. Amantadine may therefore be useful in modulating behaviour in some young patients, but it is not clear whether this is because of its glutamatergic activity or enhancement of dopaminergic neurotransmission.

\section{Clinical implications}

In children with any form of developmental disability, hyperactivity and inattention are more complex to treat than in neurotypical children: response is more idiosyncratic and unwanted effects are more common. Thus far, immediaterelease methylphenidate has the most convincing evidence base with regard to symptoms of motor hyperactivity and inattention in children and adolescents. Research involving adults with autism-spectrum disorders who experience these symptoms, as in the non-autism adult population with such problems, continues to lag far behind. Recent National Institute for Health and Clinical Excellence guidelines (National Collaborating Centre for Mental Health 2008) do not specifically mention individuals with autismspectrum disorders who also have significant problems with hyperkinesis. However, they state that drug treatments should be offered as first-line treatment only to patients with severe symptoms or impairment, and always in combination with 
psychosocial interventions. Methylphenidate, dexamfetamine and atomoxetine are all regarded as options for management.

\section{Mood disorders}

Patients with autism have been reported to be at increased risk of depression (prevalence of about $2 \%$ of all autism-spectrum disorders), particularly those who are more cognitively able (Ghaziuddin 2002). However, a recent follow-up study suggests that the incidence of mood disorder may be no different from the general population (Hutton 2008). Nevertheless, depressive symptoms remain an important treatable cause of deterioration in functioning.

Mood disorder tends to emerge from middle childhood and is often associated clinically with the child's increased self-awareness of difference and the increasing social demands of peers. As in other childhood mood disorders, psychosocial management is the first-line approach, and adaptation of the social and educational environment is often a first-line target.

For persistent and/or severe depressive disorder in children and adolescents, open-label trials of SSRIs, including fluoxetine, fluvoxamine, sertraline, citalopram and escitalopram, in children and adults have been associated with improvements in a depressive phenotype, including such symptoms as social withdrawal, irritability, sadness or crying, decreased energy and weight loss. Systematic reviews have confirmed that SSRIs should be considered for the treatment of depressive symptoms in autism (Posey 2006).

Anticonvulsants such as divalproex have been posited to be useful in mood lability (Myers 2007). Several case reports describe patients with autism and atypical bipolar disorder who responded well to open-label treatment with lithium (e.g. Kerbeshian 1987).

\section{Anxiety disorders}

Individuals with autism-spectrum disorders show increased risk of anxiety disorders (Kim 2000). It is often particularly intense and sometimes atypical; sometimes interacting with core symptomatology to even mimic thought disorder. One double-blind trial shows evidence of efficacy using fluvoxamine (McDougle 1996), and case reports of other SSRIs indicate improvements in anxiety symptoms with their use (Posey 2006).

An open-label study as well as case reports suggest that buspirone (a 5-HT agonist) may also be effective for anxiety (Buitelaar 1998a). Beta blockers may be appropriate where panic is a component symptom.

\section{Clinical implications}

Anxiety in autism-spectrum disorders has particular qualities and is often underrecognised. It can significantly increase presenting social symptomatology and social anxiety, or specific phobias can contribute to social avoidance and functional social impairment. Anxiety management strategies and desensitisation for behavioural avoidance can usefully be combined with medication management.

\section{Tic disorders and OCD}

Tourette syndrome has been shown to be comorbid in over $6 \%$ of autism cases, more than would be expected by chance (Baron-Cohen 1999). There are increasingly recognised overlaps between autismspectrum disorders, OCD and tic disorders. There is no evidence that pharmacological treatment of these disorders should differ from that used in each disorder alone. The use of haloperidol, clonidine and risperidone for tics, and clomipramine or SSRIs for obsessions and compulsions, is well described in the literature (Gringras 2000).

\section{Sleep disorders}

There is some evidence of abnormal melatonin regulation in autism (Paavonen 2003). Openlabel studies suggest melatonin may be effective for improving sleep onset, but controlled trials are lacking (Gringras 2000). Alpha-2 agonists, antihistamines, ramelteon (a melatonin receptor agonist) and mirtazapine have all been reported to have some effect on sleep in open-label trials and case reports (e.g. Owens 2005).

\section{Clinical implications}

Sleep disorder is common and often underrecognised. It is the cause of long-term debility for families and daytime symptoms in patients. Many parents are focused on medication management for these problems, and they can be effective equally important is systematic sleep hygiene and behavioural measures.

\section{Thought disorder}

There is consensus in clinical literature on the occurrence of atypical quasi-psychotic symptomatology in autism. This is often transient but can be recurring or persistent in mild form. Such symptomatology is important to recognise as it often causes diagnostic confusion and inappropriate treatment response. The nosological status of such states is not agreed: they do not necessarily represent a psychotic prodrome and the prevalence of psychosis is not markedly increased. 
The acute transient form can often be appropriately classified as a brief reactive psychosis; a useful syndromic account of the relapsing form has been given under the descriptive if inelegant term of 'multiplex complex developmental disorder' (Buitelaar 1998b).

These states can often be managed conservatively without medication by identification and reduction of relevant environmental stressors. However, the more recurrent forms will often need medication management, and the correct response here is for titrated antipsychotic medication, often with low-dose maintenance treatment to prevent a relapsing course. There are rarer states that also need consideration in a differential diagnosis, such as emerging developmental abnormalities in adolescence associated with microdeletion on 22q11 (the velocardiofacial syndrome).

\section{Discussion}

Research in many of the areas discussed is still in its early phases, and the ongoing limitations of the evidence and intrinsic difficulties of measurement are important for clinicians to remember when discussing things with often well-informed and frustrated families. Box 1 highlights some of the evidential shortcomings of published literature in this field. There are few RCTs, and trials are often small and underpowered. Long-term studies of unwanted effects are often lacking. The population studied is not homogeneous and many trials use disparate outcome measures, which makes comparisons difficult or simply invalid. Many outcome measures subsume a variety of elements of behaviour, making it hard to be clear about what the data actually show for specific areas (Wisniewski 2007).

Moreover, thereis theinherent difficulty of studying pharmacological response in developmental

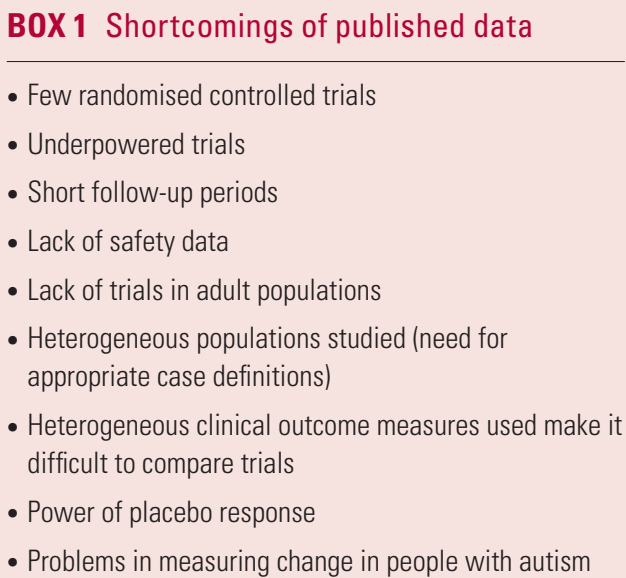

disorders themselves. Measuring change in autismspectrum disorders is complex and must take into account day-to-day fluctuations, powerful placebo effects and idiosyncratic responses. The examples of fenfluramine and secretin illustrate these particular difficulties. Reasons for a large placebo response in children with autism include heightening of positive expectancy by media attention, by sensory experiences associated with intravenous injections, and participation effects modifying caregivers' behaviour. Sandler (2005) discusses these issues in detail.

The underlying developmental trajectory of individual patients is highly variable and may confound apparent positive responses to medication. This issue is more widely recognised now and researchers (e.g. Research Units on Paediatric Psychopharmacology Autism Network 2005a,b; Scahill 2006) are attempting to address these concerns; however, the field awaits a critical mass of larger, more robust clinical trials with more specific measurements and sophisticated analyses of clinical effectiveness. Clinicians will need to carefully interpret the clinical evidence and make active adjustments in applying it to the specific situation of their patients.

Despite the limitations, however, both the quantity and quality of medication trials targeting symptom domains in autism-spectrum disorders have increased in recent years. There remains a lack of important information on long-term safety and efficacy of drugs, and the standard of evidence so far does not allow for definitive treatment protocols for various symptom clusters. However, the aggregation of data suggests that SSRIs, risperidone and immediate-release methylphenidate can be of great value within the domains discussed. There are as yet no proven treatments for the underlying social deficit in autism.

We have emphasised that medication management is only one strand of interventions for people with autism and the mainstay of treatment remains educational and psychosocial. Pharmacological management needs to be undertaken by thorough assessment, accurate diagnosis and regular monitoring of target symptom clusters, comorbid diagnoses and response to treatment (Box 2).

\section{Looking to the future}

There are several exciting new developments within the field of pharmacology research in autismspectrum disorders. Large trials in glutamatergic function are currently ongoing, particularly in the USA. Most studies to date focus on the use of one drug to target one group of related symptoms. One such study is an ongoing RCT of aripiprazole $v$. 
B0X 2 Important considerations when prescribing for autism

To make informed decisions about a potential role for medication, the prescriber must:

- clarify characteristics of the challenging behaviours including frequency, intensity, duration and degree of interference with functioning

- be clear about the target symptomatology to be treated (differentiate core from comorbid symptoms)

- identify exacerbating and ameliorating factors, including response to psychotherapeutic interventions
- assess existing and available health, educational and social supports, and the strengths of the family (e.g. the family's ability to support the individual)

- assess comorbid physical problems by thorough history and examination, and consider their impact on presentation and treatment of the challenging behaviours

- consider potential adverse events and drug interactions

- avoid polypharmacy

- use a 'start low and go slow' treatment strategy. placebo in the reduction of aggressive and aberrant behaviour in children with autism (principal investigator: S. L. Novotny; http://clinicaltrials. gov/ct2/show/NCT00468130). Studies using more than one drug to target more than one symptom domain (so called 'coactive studies') are also being considered in treatment trials: D-cycloserine and aripiprazole are currently the subjects of a large trial to target both social impairment and the domain of aggression/self-injury (McDougle 2006). Researchers are beginning to consider the value of a more formal combination of behavioural and medical interventions in complex treatment trials designed to alter the developmental trajectory of those with autism. Advances in neurophysiology and genetics may also make it possible to delineate subgroups that may be particularly responsive to particular treatments. Such developments may pave the way for a more integrated consensus on an overall approach to treatment of autism-spectrum disorders across the lifespan.

\section{References}

Aman MG, Lam KSL, Van Bourgondien ME (2005) Medication patterns in patients with autism: temporal, regional, and demographic influences. Journal of Child and Adolescent Psychopharmacology 15: $116-26$

Arnold LE, Aman MG, Cook AM, et al (2006) Atomoxetine for hyperactivity in autism spectrum disorders: placebo-controlled crossover pilot trial. Journal of the American Academy of Child and Adolescent Psychiatry 45: 1196-205

Autism Genome Project Consortium (2007) Mapping autism risk loci using genetic linkage and chromosomal rearrangements. Nature Genetics 39 : 319-28.

Autism Speaks (2009) Autism Speaks announces results reported for the study of fluoxetine in autism (SOFIA). Autism Speaks (http://www. autismspeaks.org/press/as_announces_sofia_results.php).

Baird G, Simonoff E, Pickles A, et al (2006) Prevalence of disorders of the autism spectrum in a population cohort of children in South Thames: the Special Needs and Autism Project (SNAP). Lancet 368: 210-5.
Baron-Cohen S, Mortimore C, Moriarty J, et al (1999) The prevalence of Gilles de la Tourette's syndrome in children and adolescents with autism. Journal of Child Psychology and Psychiatry 40: 213-8.

Belsito KM, Law PA, Kirk KS, et al (2001) Lamotrigine therapy for autistic disorder: a randomized, double-blind, placebo-controlled trial. Journal of Autism and Developmental Disorders 31: 175-81.

Broadstock M, Doughty C, Eggleston M (2007) Systematic review of the effectiveness of pharmacological treatments for adolescents and adults with autism spectrum disorder. Autism 11: 335-48.

Buchsbaum MS, Hollander E, Haznedar MM, et al (2001) Effect of fluoxetine on regional cerebral metabolism in autistic spectrum disorders: a pilot study. International Journal of Neuropsychopharmacology 4 : $119-25$

Buitelaar JK, van der Gaag RJ, van der Hoeven J (1998a) Buspirone in the management of anxiety and irritability in children with pervasive developmental disorders: results of an open-label study. Journal of Clinical Psychiatry 59: 56-9.

Buitelaar J, van der Gaag R (1998b) Diagnostic rules for children with PDDNoS and multiple complex developmental disorder. Journal of Child Psychology and Psychiatry 39: 911-9.

Carlsson ML (1998) Hypothesis: is infantile autism a hypoglutamatergic disorder? Relevance of glutamate-serotonin interactions for pharmacotherapy. Journal of Neural Transmission 105: 525-35.

Chez MG, Aimonovitch M, Buchanan T, et al (2004) Treating autistic spectrum disorders in children: utility of the cholinesterase inhibitor rivastigmine tartrate. Journal of Child Neurology 19: 165-9.

Davanzo PA, Belin TR, Widawski MH, et al (1998) Paroxetine treatment of aggression and self-injury in persons with mental retardation. American Journal on Mental Retardation 102: 427-37.

Dunbar F, Kusumakar V, Daneman D, et al (2004) Growth and sexual maturation during long-term treatment with risperidone. American Journal of Psychiatry 161: 918-20.

Dykens EM (2000) Annotation: psychopathology in children with intellectual disability. Journal of Child Psychology and Psychiatry and Allied Disciplines 41: 407-17

Erickson CA, Posey DJ, Stigler KA, et al (2007) Pharmacologic treatment of autism and related disorders. Pediatric Annals 36: 575-85.

Fankhauser MP, Karumanchi VC, German ML, et al (1992) A doubleblind, placebo-controlled study of the efficacy of transdermal clonidine in autism. Journal of Clinical Psychiatry 53: 77-82.

Feldman HM, Kolmen BK, Gonzaga AM (1999) Naltrexone and communication skills in young children with autism. Journal of the American Academy of Child and Adolescent Psychiatry 38: 587-93.

Fombonne $\mathrm{E}$ (2003) The prevalence of autism. JAMA 289: 87-9.

Fombonne $E$ (2005) The changing epidemiology of autism. Journal of Applied Research in Intellectual Disabilities 18: 281-94.

Ghaziuddin M, Ghaziuddin N, Greden J (2002) Depression in persons with autism: implications for research and clinical care. Journal of Autism and Developmental Disorders 32: 299-306

Gringras P (2000) Practical paediatric psychopharmacological prescribing in autism: the potential and the pitfalls. Autism 4: 229-47.

Harrigan EP, Miceli JJ, Anziano R, et al (2004) A randomized evaluation of the effects of six antipsychotic agents on OTc, in the absence and presence of metabolic inhibition. Journal of Clinical Psychopharmacology 24: $62-9$.

Hellings JA, Weckbaugh M, Nickel EJ, et al (2005) A double-blind, placebo-controlled study of valproate for aggression in youth with pervasive developmental disorders. Journal of Child and Adolescent Psychopharmacology 15: 682-92.

Hollander E, Kaplan A, Cartwright C, et al (2000) Venlafaxine in children, adolescents, and young adults with autism spectrum disorders: an open retrospective clinical report. Journal of Child Neurology 15: 132-5.

Hollander E, Dolgoff Caspar R, Cartwright C, et al (2001) An open trial of divalproex sodium in autism spectrum disorders. Journal of Clinical Psychiatry 62: 530-4. 
Hollander E, Phillips A, Chaplin W, et al (2005) A placebo controlled crossover trial of liquid fluoxetine on repetitive behaviors in childhood and adolescent autism. Neuropsychopharmacology 30: 582-9.

Hollander E, Wasserman S, Swanson EN, et al (2006a) A double-blind placebo-controlled pilot study of olanzapine in childhood/adolescent pervasive developmental disorder. Journal of Child and Adolescent Psychopharmacology 16: 541-8.

Hollander E, Soorya L, Wasserman S, et al (2006b) Divalproex sodium vs. placebo in the treatment of repetitive behaviours in autism spectrum disorder. International Journal of Neuropsychopharmacology 9: 209-13.

Honda H, Shimizu Y, Rutter M (2005) No effect of MMR withdrawal on the incidence of autism: a total population study. Journal of Child Psychology and Psychiatry 46: 572-9.

Hutton J, Goode S, Murphy M, et al (2008) New-onset psychiatric disorders in individuals with autism. Autism 12: 373-90.

Ing BH, Wright DM, Handen BL, et al (2001) Double blind, placebo controlled study of amantadine hydrochloride in the treatment of children with autistic disorder. Journal of the American Academy of Child and Adolescent Psychiatry 40: 658-65.

Jaselskis CA, Cook EH, Fletcher KE, et al (1992) Clonidine treatment of hyperactive and impulsive children with autistic disorder. Journal of Clinical Psychopharmacology 12: 322-7

Jahromi LB, Kasari CL, McCracken JT, et al (2009) Positive effects of methylphenidate on social communication and self-regulation in children with pervasive developmental disorders and hyperactivity. Journal of Autism and Developmental Disorders 39: 395-404.

Jesner OS, Aref-Adib M, Coren E (2007) Risperidone for autism spectrum disorder. Cochrane Database of Systematic Reviews issue 1: CD005040.

Kerbeshian J, Burd L, Fisher W (1987) Lithium carbonate in the treatment of two patients with infantile autism and atypical bipolar symptomatology. Journal of Clinical Psychopharmacology 7: 401-5.

Kim JA, Szatmari P, Bryson SE, et al (2000) The prevalence of anxiety and mood problems among children with autism and Asperger syndrome. Autism 4: 117-32.

King BH, Wright DM, Handen BL, et al (2001) Double-blind, placebocontrolled study of amantadine hydrochloride in the treatment of children with autistic disorder. Journal of the American Academy of Child and Adolescent Psychiatry 40: 658-65.

King BH, Hollander E, Sikich L, et al (2009) Lack of efficacy of citalopram in children with autism spectrum disorders and high levels of repetitive behavior: citalopram ineffective in children with autism. Archives of General Psychiatry 66: 583-90.

Lecavalier L, Leone S, Wiltz J (2006) The impact of behaviour problems on caregiver stress in young people with autism spectrum disorders. Journal of Intellectual Disability Research 50: 172-83.

Lee D0, Ousley OY (2006) Attention-deficit hyperactivity disorder symptoms in a clinic sample of children and adolescents with pervasive developmental disorders. Journal of Child and Adolescent Psychopharmacology 16: 737-46.

Lord C, Leventhal BL, Cook EH (2001) Quantifying the phenotype in autism spectrum disorders. American Journal of Medical Genetics Part B: Neuropsychiatric Genetics 105: 36-8.

Luby J, Mrakotsky C, Stalets MM, et al (2006) Risperidone in preschool children with autistic spectrum disorders: an investigation of safety and efficacy. Journal of Child and Adolescent Psychopharmacology 16: $575-87$

Martin A, Koenig K, Scahill L, et al (1999) Open-label quetiapine in the treatment of children and adolescents with autistic disorder. Journal of Child and Adolescent Psychopharmacology 9: 99-107.

McDougle CJ, Naylor ST, Cohen DJ, et al (1996) A double-blind, placebocontrolled study of fluvoxamine in adults with autistic disorder. Archives of General Psychiatry 53: 1001-8.

McDougle CJ, Holmes JP, Carlson DC, et al (1998) A double blind placebo controlled study of risperidone in adults with autistic disorder and other pervasive developmental disorders. Archives of General Psychiatry 55: $633-41$
McDougle CJ, Kresch LE, Posey DJ (2000) Repetitive thoughts and behavior in pervasive developmental disorders: treatment with serotonin reuptake inhibitors. Journal of Autism and Developmental Disorders 30: $427-35$

McDougle CJ, Scahill L, Aman MG, et al (2005) Risperidone for the core symptom domains of autism: results from the study by the Autism Network of the Research Units on Pediatric Psychopharmacology. American Journal of Psychiatry 162: 1142-8.

McDougle CJ, Stigler KA, Erickson CA, et al (2006) Pharmacology of autism. Clinical Neuroscience Research 6: 179-88.

Mesibov GB, Handlan S (1997) Adolescents and adults with autism. In Handbook of Autism and Pervasive Developmental Disorders (eds DJ Cohen, FR Volkman): 309-22. John Wiley \& Sons.

Myers SM (2007) The status of pharmacotherapy for autism spectrum disorders. Expert Opinion in Pharmacotherapy 8: 1579-603.

National Collaborating Centre for Mental Health (2008) Attention Deficit Hyperactivity Disorder: Diagnosis and Management of ADHD in Children, Young People and Adults (National Clinical Practice Guideline Number 72). National Institute for Health and Clinical Excellence.

National Initiative for Autism: Screening and Assessment (2003) National Autism Plan for Children (NAPC). Plan for the Identification, Assessment, Diagnosis and Access to Early Interventions for Pre-school and Primary School Aged Children with Autism Spectrum Disorders (ASD). National Autistic Society (http://iier.isciii.es/autismo/pdf/aut_napc.pdf).

Neiderhofer H, Staffer W, Mair A (2002) Lofexidine in hyperactive and impulsive children with autistic disorder. Journal of the American Academy of Child and Adolescent Psychiatry 41: 1396-7.

Nikolov R, Jonker J, Scahill L (2006) Autistic disorder: current psychopharmacological treatments and areas of interest for future developments [Portuguese, English]. Revista Brasileira de Psiquiatria 28 (suppl 1): S39-46.

Nye C, Brice A (2005) Combined vitamin B6-magnesium treatment in autism spectrum disorder. Cochrane Database of Systematic Reviews issue 4: CD003497.

Owens JA, Babcock D, Blumer J, et al (2005) The use of pharmacotherapy in the treatment of pediatric insomnia in primary care: rational approaches. A consensus meeting summary. Journal of Clinical Sleep Medicine 1: 49-59.

Owley T, Walton L, Salt J, et al (2005) An open-label trial of escitalopram in pervasive developmental disorders. Journal of the American Academy of Child and Adolescent Psychiatry 44: 343-8.

Paavonen EJ, Nieminen-von Wendt T, Vanhala R, et al (2003) Effectiveness of melatonin in the treatment of sleep disturbances in children with Asperger disorder. Journal of Child and Adolescent Psychopharmacology 13: 83-95.

Panksepp J, Lensing P (1991) A synopsis of an open-trial of naltrexone treatment of autism with four children. Journal of Autism and Developmental Disorders 21: 243-9.

Posey DJ, Kem DL, Swiezy NB, et al (2004) A pilot study of D-cycloserine in subjects with autistic disorder. American Journal of Psychiatry 161: 2115-7.

Posey DJ, Erickson CA, Stigler KA, et al (2006) The use of selective serotonin reuptake inhibitors in autism and related disorders. Journal of Child and Adolescent Psychopharmacology 16: 181-6.

Posey DJ (2008) A double-blind placebo-controlled study of D-cycloserine in children with autistic disorder. In Proceedings of the 55th Annual Meeting of the American Academy of Child and Adolescent Psychiatry: Abstract 3.53. AACAP.

Quintana H, Birmaher B, Stedge D, et al (1995) Use of methylphenidate in the treatment of children with autistic disorder. Journal of Autism and Developmental Disorders 25: 283-94.

Remington G, Sloman L, Konstantareas M, et al (2001) Clomipramine versus haloperidol in the treatment of autistic disorder: a doubleblind, placebo-controlled, crossover study. Journal of Clinical Psychopharmacology 21: 440-4.
MCO answers

1 b $\quad 2$ c $\quad 3$ e $\quad 4$ a $5 c$ 
Research Units on Paediatric Psychopharmacology Autism Network (2005a) Randomised, controlled, crossover trial of methylphenidate in pervasive developmental disorders with hyperactivity. Archives of General Psychiatry 62: 1266-74

Research Units on Paediatric Psychopharmacology Autism Network (2005b) Risperidone treatment of autistic disorder: longer term benefits and blinded discontinuation after 6 months. American Journal of Psychiatry 162: 1361-9.

Sandler RH, Finegold SM, Bolte ER, et al (2000) Short-term benefit from oral vancomycin treatment of regressive-onset autism. Journal of Child Neurology 15: 429-35

Sandler A (2005) Placebo effects in developmental disabilities: implications for research and practice. Mental Retardation and Developmental Disabilities Research Reviews 11: 164-70.

Santosh PJ, Baird G, Pityaratstian N, et al (2006) Impact of comorbid autism spectrum disorders on stimulant response in children with attention deficit hyperactivity disorder: a retrospective and prospective effectiveness study. Child: Care, Health and Development 32: 575-83.

Scahill L, McCracken JT, McGough J, et al (2002) Risperidone in children with autism and serious behavioral problems. New England Journal of Medicine 347: 314-21.

Scahill L, Aman MC, McDougle CJ, et al (2006) A prospective open trial of guanfacine in children with pervasive developmental disorders. Journal of Child and Adolescent Psychopharmacology 16: 589-98.

Shea S, Turgay A, Carroll A, et al (2004) Risperidone in the treatment of disruptive behavioral symptoms in children with autistic and other pervasive developmental disorders. Pediatrics 114: e634-41.
Stigler KA, Desmond LA, Posey DJ, et al (2004) A naturalistic retrospective analysis of psychostimulants in pervasive developmental disorders. Journal of Child and Adolescent Psychopharmacology 14: 49-56.

Stigler KA, Diener JT, Kohn AE, et al (2009) Aripiprazole in pervasive developmental disorder not otherwise specified and Asperger's disorder: a 14-week, prospective, open-label study. Journal of Child and Adolescent Psychopharmacology 19: 265-74.

Sugie $Y$, Sugie H, Fukuda T, et al (2005) Clinical efficacy of fluvoxamine and functional polymorphism in a serotonin transporter gene on childhood autism. Journal of Autism and Developmental Disorders 35 . $377-85$

Symons FJ, Thompson A, Rodriguez MC (2004) Self injurious behaviour and the efficacy of naltrexone treatment: a quantitative synthesis. Mental Retardation and Developmental Disability Research Review 10 : 193-200.

Tsakanikos E, Costello H, Holt G, et al (2006) Psychopathology in adults with autism and intellectual disability. Journal of Autism and Developmental Disorders 36: 1123-9.

Wisniewski, T, Brimacombe MB Ming X (2007) Pharmaceutical treatment studies in autism and ADHD: a design based review of study quality. Journal of Pediatric Neurology 5: 189-97.

World Health Organization (1994) ICD-10 Classification of Mental and Behavioural Disorders. WHO.

Yurgelun-Todd DA, Coyle JT, Gruber SA, et al (2005) Functional magnetic resonance imaging studies of schizophrenic patients during word production: effects of D-cycloserine. Psychiatry Research 138 . 23-31.

\section{MCQs}

Select the single best option for each question stem

1 Selective serotonin reuptake inhibitors:

a are of proven efficacy in the treatment of core social impairment in autism

b may cause behavioural activation in children with autism

c are not indicated for the treatment of obsessions and compulsions in autismspectrum disorders

d have been demonstrated to be safe in the longterm treatment of patients with autism

e are not indicated for the treatment of interfering ritualised behaviours in adolescents with autism.

2 Which of the following is considered an effective treatment for anxiety symptoms in autism-spectrum disorders?

a methylphenidate

b risperidone

\section{c buspirone}

d D-cycloserine

e vitamin B6.

3 Which of the following is considered an effective treatment for aggressive and/or self-injurious behaviour in autism?

a quetiapine

b clomipramine

c methylphenidate

d atomoxetine.

e risperidone.

\section{Risperidone:}

a may lead to clinically significant increases in prolactin levels in patients with autism

$\mathrm{b}$ is of proven safety in the treatment of behaviours associated with autism in the elderly population

c is never indicated in the treatment of aggression and severe self-harm in patients with autism d is considered an effective treatment for depressive symptoms

e always leads to significant QTc prolongation in ECGs of children with autism.

5 Research evidence shows that:

a the ratio of male:female patients with autismspectrum disorders is 8:1

b the prevalence of narrowly defined autism is 2 per 1000 children

c up to $75 \%$ of people with autism are prescribed at least one psychotropic medication in the UK

$\mathrm{d}$ children with autism and hyperkinetic symptoms do not respond to immediate-release methylphenidate preparations

e diagnostic criteria for psychiatric disorders do not have to be modified in patients with autism and intellectual disability. 